\title{
La colección de microalgas de la Universidad Pedagógica Nacional como estrategia didáctica para la enseñanza y aprendizaje de conceptos biológicos
}

\author{
Collection of Microalgae as the National Pedagogical University \\ Teaching strategies for teaching and learning of biological concepts \\ Ibeth Delgadillo, Femando Góngora y Francisco Medellín
}

Universidad Pedagógica Nacional. ibepadero@yahoo.com, femichur@gmail.com, franciscomedellin@hotmail.com

\section{Resumen}

Debido a la complejidad en la construcción de algunos conceptos biológicos y aunque los docentes recurren a diversas formas didácticas, existen carencias de recursos que dinamicen los procesos de enseñanza-aprendizaje; es por esto que surge el trabajo de grado que propone la utilización de la Colección de microalgas para fortalecer y aumentar la calidad de los procesos educativos, basandose en el principio didáctico que indica que "se aprende más a través de la participación activa, enfocándose el interés en aprender haciendo" (Segura, 2000). Para la reestructuración de la Ficoteca de la Universidad Pedagógica Nacional, se elaboraron materiales educativos que transforman la colección en una estrategia didáctica, que al ser utilizada en el aula de dase permite que los alumnos aprendan por su propia experiencia, encaminándose en el descubrimiento de las ideas; como explica Segura (2000), "no constituye un medio para facilitar la enseñanza, sino que es la enseñanza misma, ya que manipular es aprender".

Palabras clave

Colección biológica, Ficoteca, Enseñanza-Aprendizaje, Aprendizaje Significativo, Estrategia Didáctica, Microalgas.

\section{Abstract}

Due to the complexity in the construction of some biological concepts and although the educational ones resorts to diverse didactic forms, exist deficiencies of resources that dinamicen the education-learning processes; it is by that the work arises from degree that proposes the use of the Collection of seaweed to fortify and to increase the quality of the educative processes, being based on the didactic principle that it indicates that "more through the active participation is learned, focusing the interest in learning doing" (Segura, 2000). For the reconstruction of the Ficoteca of the National University Pedagogical, they were elaborated material educative which they transform the collection into a didactic strategy, that to the being used in the dass classroom allows that the students leam by their own experience, directing itself in the discovery of the ideas; as it explains Segura (2000), "it does not constitute an average one to facilitate education, but that is same education, since to manipulate it is to learn".

Keywords

Biological Collection, Ficoteca, Education-Learning, Significant Learning, Didactic Strategy, Seaweed. 


\section{Introducción}

En la continua búsqueda de elementos didácticos que permitan desenvolvernos en nuestro papel de docentes, el Departamento de Biología (DBI) de la Universidad Pedagógica Nacional (UPN), ha producido diferentes colecciones biológicas que en algunos casos son utilizadas como ayuda en los procesos de enseñanza-aprendizaje de las ciencias; sin embargo, colecciones como la Ficoteca han sido relegadas, desaprovechando su carácter de contribución en la educación, por falta de organización, herramientas o simplemente interés de los mismos usuarios. Es así como surge la siguiente situación problema, que permite el diseño y elaboración del presente trabajo: ¿Cómo potenciar los procesos de enseñanza-aprendizaje de conceptos biológicos generados en el Departamento de Biología, a través de la reestructuración de la Colección de Microalgas (Ficoteca) de la Universidad Pedagógica Nacional como estrategia didáctica?

Para tal fin, se establecen objetivos espeáficos buscando:

1) Facilitar el uso de la colección de microalgas en los procesos académicos del Departamento de Biología, mediante la curaduría de las muestras que se encuentran en la Ficoteca de la Universidad Pedagógica Nacional.

2) Fortalecer el uso de la colección desde el diseño y elaboración de una guía ilustrada y una base de datos donde se compile información relacionada con el estudio de las Microalgas.

3) Proporcionar apoyo y servicio a las actividades de docencia, investigación y difusión que se realizan en el DBI, a partir de los materiales educativos (Micropreparados, guía ilustrada, cartilla de métodos y la galería fotográfica) generados en la reestructuración de la Ficoteca.

Dentro de los resultados se pudo encontrar, que existe la necesidad de generar continuamente materiales educativos y actividades complementarias para el aula de dase, en este sentido se demuestra a través del instrumento aplicado que las colecciones biológicas generan un interés en los estudiantes desde el carácter investigativo, como de carácter educativo y procedimental. Por otra parte, se demuestra que la colección de microalgas en términos de su utilización para la docencia, debe establecer una estructura y organización que le permita brindar los medios para ser utilizada como una estrategia didáctica dentro del aula de dases.

\section{Metodología}

A partir de los materiales existentes en la colección de microalgas se realizaron diversas actividades articuladas de manera que permitieran constituir una colección biológica accequible y utilizable por docentes y estudiantes del Departamento de Biología de la UPN así como de otras instituciones interesadas, para ello se desarrollaron las siguientes fases:

Fase de diagnóstico: Se realiza el diseño, validación, aplicación, tabulación y análisis de un instrumento constituido por una encuesta diagnóstico aplicado a 180 estudiantes y 12 docentes del DBI. El análisis de las encuestas se guió por Baeza (2002), partiendo de la tabulación de las preguntas de selección múltiple y para las preguntas abiertas se utilizó la categorización según Josep \& Hilferty (1999).

Fase de curaduría: Se establece un grupo de muestras de docencia y otro de referencia y este último en grupos por las diferentes provincias Limnológicas propuestas por Donato (1991). Se determinan las microalgas hasta género con las daves de Biaudo \& Menezes (2006), Parra, et al. (1982), Ramírez (2000), entre otros.

La información obtenida de la curaduría se sistematiza mediante una base de datos en Access 2007, la cual contiene información de cada muestra clasificada en campos referentes al ingreso, colecta, biología, imágenes y micropreparados. 
Fase de elaboración de material educativo: Durante esta fase se generan una serie de materiales educativos provenientes del proceso de curaduría y la compilación de documentos que permitan un acercamiento a las microalgas, produciendo así una guía ilustrada, cartilla de métodos, Micropreparados, y una galería fotográfica.

Fase de aplicación y validación de la estrategia: Con el fin de legitimar que la Colección de Microalgas es una estrategia didáctica para la enseñanza y aprendizaje de conceptos biológicos dentro del Departamento de Biología, se diseñó un instrumento que permitiera la evaluación de los materiales educativos generados a partir de la reestructuración de la Ficoteca; además se generó una actividad donde se pusiera a prueba el papel de la estrategia didáctica, dentro de la enseñanza y aprendizaje de una temática espećfica.

\section{Discusión}

Encuesta diagnóstico: La encuesta permitió establecer que de los 180 estudiantes encuestados del Departamento de Biología en el primer periodo del 2008, como lo muestra la gráfica № 1, la tendencia más alta remite a la categoría de material de apoyo como muestras /espeámenes, demostrando que la mayoría de los estudiantes del DBI ven las colecciones en función de las prácticas de laboratorio, teniendo a los espećmenes como los elementos más importantes y a su vez como el principal servicio que debe prestar una colección biológica. Sin embargo es de rescatar que el segundo servicio más mencionado en las encuestas, es el de carácter educativo, visto como sustento y apoyo pedagógico o didáctico para las actividades académicas que se desarrollan dentro del aula de clases.

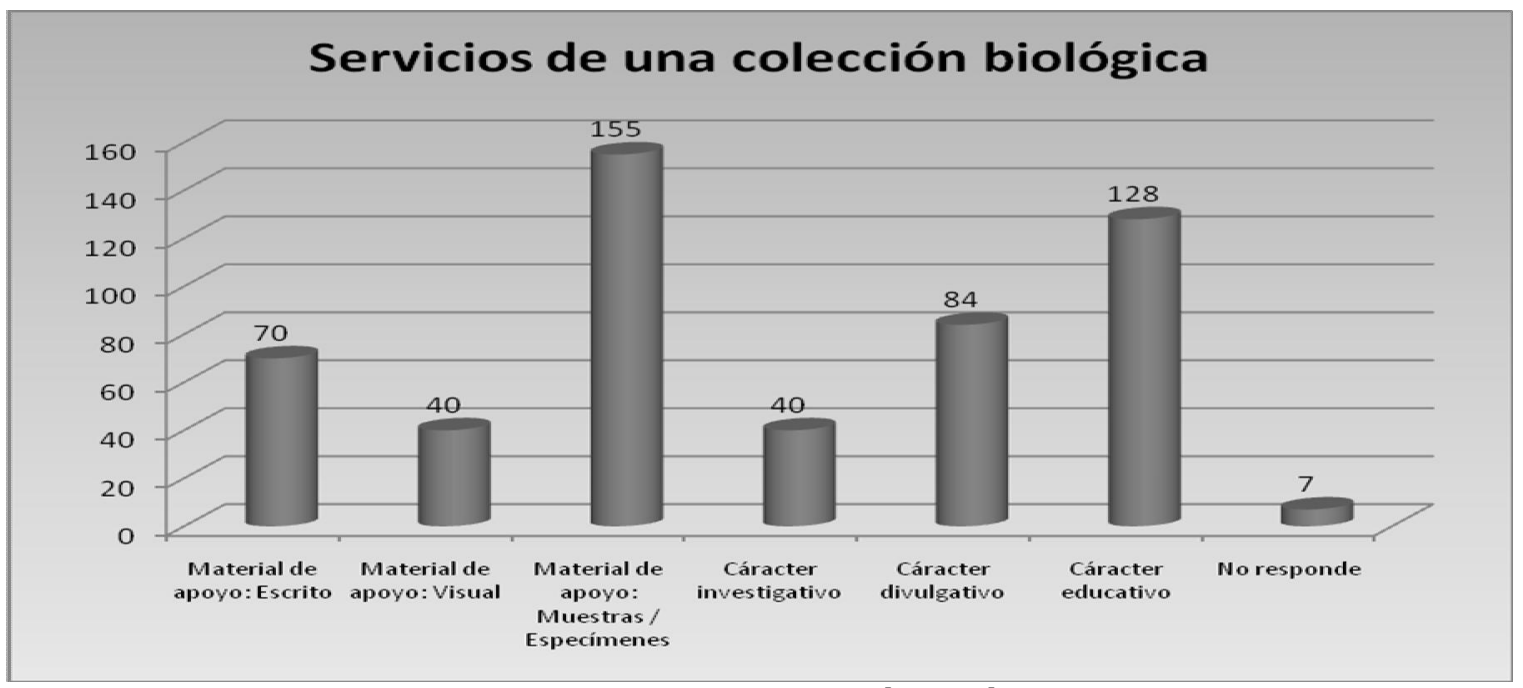

Figura No. 1. Servicios de una colección biológica UPN.

La gama de posibilidades para trabajar con una colección biológica son infinitas, para el caso de la colección de microalgas de la Universidad Pedagógica Nacional, el apoyo en actividades académicas presenta el mayor número de respuestas, ya que el facilitar los procesos educativos dentro del aula es resaltado como una de las necesidades primordiales dentro del departamento de biología, teniendo en cuenta que la ficoteca puede llegar a proporcionar material de apoyo importante para realizar las practicas de laboratorio pertinentes que facilitan la comprensión de algunos conceptos biológicos mediante la observación directa. De igual forma los proyectos investigativos permiten deducir un amplio interés por promover la investigación dentro del DBI y 
muestra que junto con las prácticas de reconocimiento, son opciones que los estudiantes tienen en auenta a la hora de trabajar con microalgas.

De otro lado, se trasciende en la mirada de las colecciones como espacios de almacenamiento de espeámenes y de investigación netamente cient́fica, para dar paso a una mirada holística donde se comprende la colección biológica como un eje articulador que promueve y potencializa los procesos educativos dentro y fuera del aula, ya que tiene en cuenta la enseñanza de contenidos tanto conceptuales como procedimentales y actitudinales, esto reflejado en las respuestas que tienen en cuenta el manejo de colecciones, mostrado en la grafica № 3.

\section{Posibilidades de trabajo con la Ficoteca}

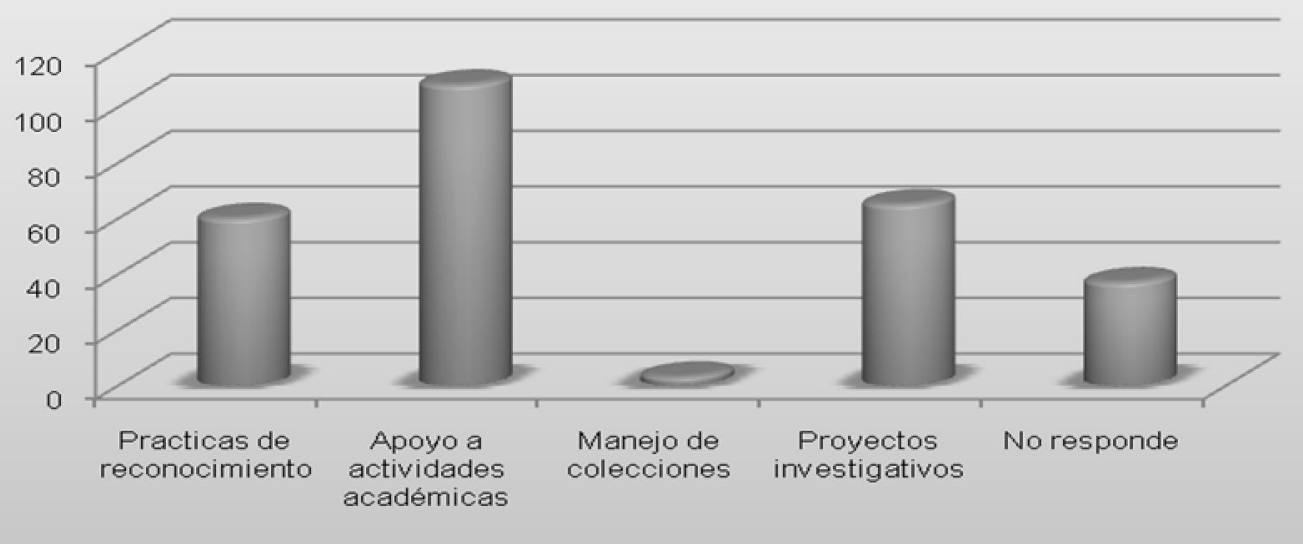

Figura No. 2. Posibilidades de trabajo con la Ficoteca del DBI

Curaduría: En el proceso de curaduría de la colección de microalgas se encontraron 285 muestras de las cuales 148 tenían etiquetas con información de colecta, y 137 no contaban con ningún tipo de información; así se dasifican en muestras de Referencia y de Docencia respectivamente.

Del total de las muestras encontradas al principio, se lograron recuperar 60 muestras de Docencia y 70 de Referencia. Entre los géneros más representativos que se encontraron en las muestras de la Ficoteca se pueden destacar: Anabaena, Aulacoseira, Chlorococaum, Closterium, Coelastrum, Cosmarium, Euglena, Melosira, Microcystis, Naviaula, Phacus, Oscillatoria, Pinnularia, Scenedesmus, Staurodesmus, Staurastrum, Synedra, y Traquelomona.

Materiales educativos: En el proceso de elaboración de los materiales educativos se tuvieron en cuenta las necesidades que expresaron tanto estudiantes como maestros dentro de la encuesta diagnóstico; las cuales fueron enfocadas en materiales como la guía ilustrada, la cartilla de métodos, los micropreparados, la organización de las claves y la generación de una galería fotográfica; que permitieran un acceso directo a la colección y la utilización de la misma como apoyo a las diferentes actividades académicas (dases, prácticas de laboratorio, talleres, investigaciones, etc.) que se desarrollan en el DBI. Además se realizó la evaluación de didhos materiales con el fin de establecer sus fortalezas y debilidades en el momento de hacer uso de ellos en el aula de clase.

Validación de la estrategia didáctica: Dentro de esta gama de posibilidades, se hace efectiva la validación con una dase teórico-práctica entomo al macro concepto de célula, planteada a partir de la Ficoteca donde se induyen las microalgas como objeto de estudio y los materiales educativos como apoyo para trabajar temáticas referidas a la definición de célula, organelos, 
diversidad de formas, taxonomía, niveles de organización y adaptaciones morfológicas. En este sentido, se evidencia a partir de la observación, que para los estudiantes es motivante, interesante, y comprensible trabajar la temática desde la articulación de la dase magistral, la práctica de laboratorio, la orientación por parte del docente y el apoyo de una estrategia didáctica.

\section{Conclusiones}

Mediante la encuesta diagnóstico se pudo determinar la importancia que representan las colecciones biológicas dentro del departamento de biología en cuanto a su adecuado mantenimiento, con el fin de poder ser utilizadas como soporte en las diferentes actividades académicas que se desarrollan dentro de la universidad, en este sentido, se resalta el trabajo con Microalgas que se ha venido realizando y que se puede enriquecer a partir de una Ficoteca organizada y que cuente con materiales adecuados para tal fin.

En la curadunía se estableció que la colección auenta con una representatividad del $56 \%$ de los organismos algales bioindicadores reportados para Colombia por Pinilla (1998), y un 64\% de los géneros indicados por Duque \& Núñez (2000) para la Amazonía colombiana; lo que da cuenta del valor que tiene la colección en cuanto a la diversidad de

Microalgas que contiene, sin dejar de lado la necesidad de seguir realizando muestreos adecuados que enriquezcan y mejoren las condiciones en las que se encuentran las muestras.

Es de gran importancia que las colecciones biológicas quenten con la sistematización apropiada de los datos que ellas generan, además de orientación en cuanto a los aspectos más relevantes de su objeto de estudio, esto reflejado en la Ficoteca desde el diseño y elaboración tanto de la guía ilustrada, como de la base de datos, que logra proporcionar a los usuarios acceso a la información de la colección para un uso adecuado y constante de la misma.

Durante la reestructuración de la Ficoteca, se brindó apoyo a estudiantes y docentes en diversas actividades académicas y de investigación, enriquecido por la generación de materiales educativos como la guía ilustrada, la cartilla de métodos, la galería fotográfica digitalizada y micropreparados, que constituyen la enseñanza en sí mismos, ya que manipular es aprender y la mejor manera de hacerlo es con orientación y motivación.

En la validación, el mapa conceptual no es el producto final del aprendizaje, sino una expresión del significado y la importancia que cobra la Colección de Microalgas como una estrategia didáctica dentro de la enseñanza y aprendizaje de conceptos biológicos, lo que se argumenta al evidenciar que los estudiantes alcanzan una comprensión inicial del concepto célula trabajado a partir de la estrategia didáctica; esto sin dejar de lado la posibilidad de enseñar otros conceptos biológicos a partir de las Microalgas como objeto de estudio y la Ficoteca como apoyo.

Finalmente, es importante resaltar que todos los materiales educativos generados a partir del trabajo de grado son un conjunto que constituye la colección de microalgas, entendida como estrategia didáctica, una unidad dispuesta a generar retroalimentación en los procesos educativos, pero sobretodo, un conglomerado de posibilidades para facilitar que tanto el estudiante como el docente compongan un ambiente de trabajo adecuado para que los procesos de enseñanzaaprendizaje, surjan naturalmente, favoreciendo la formación integral y el compartir los conocimientos desde ambas partes.

Bibliografia

Baeza, M. (2002). De las metodologías avalitativas en investigación científico social. Chile: Universidad de Chile. 
Bicudo, C. y Menezes, M. (2006). Gêneros de algas de aguas continentais do Brasil Chave para identificação e descricỗes. Segunda Edição. RIMA Editora. São Carlos.

Bryant, J. M. (1983). Colecciones Biológicas: Legado o responsabilidad?. Curador. 3 (26).

Donato, J. (1991). Los sistemas acuáticos de Colombia: Síntesis y Revisión. Cuadernos divulgativos No 4. Colombia: Pontificia Universidad Javeriana.

Duque, S y Núñez, M. (2000). Microalgas acuáticas de la Amazonía colombiana. Revista Biota Colombiana. 1 (2), $208-216$.

Munguía, L. (2005). Estrategias didácticas. México: Colegio de ciencias y humanidades, SEMADI.

Ontoria, A. Ballesteros, A. Cuevas, C. Giraldo, L. Martin, I. Molina, A. Rodríguez, A. y Vélez, U. (1999). Mapas conceptuales: una técnica para aprender: Madrid, España: Editorial Narcea.

Pinilla, G. (1998). Indicadores biológicos en ecosistemas acuáticos continentales de Colombia.

Ramírez, J. (2000). Fitoplancton de agua dulce: Aspectos ecológicos, taxonómicos y sanitarios. Colombia: Editorial Universidad de Antioquia.

Segura, D. (2000). Constructivismo, ¿Construir qué?. Bogotá, Colombia: Escuela Pedagógica Experimental. 